\title{
Chromophobe hepatocellular carcinoma with abrupt anaplasia: a proposal for a new subtype of hepatocellular carcinoma with unique morphological and molecular features
}

Laura D Wood $^{1}$, Christopher M Heaphy ${ }^{1}$, Hubert Darius-J Daniel ${ }^{1}$, Bita V Naini ${ }^{2}$, Charles R Lassman ${ }^{2}$, May R Arroyo ${ }^{3}$, Ihab R Kamel ${ }^{4}$, David P Cosgrove ${ }^{5}$, John K Boitnott ${ }^{1}$, Alan K Meeker ${ }^{1}$ and Michael S Torbenson ${ }^{1}$

${ }^{1}$ Department of Pathology, Johns Hopkins University School of Medicine, Baltimore, MD, USA; ${ }^{2}$ Department of Pathology, University of California Los Angeles Medical Center, Los Angeles, CA, USA; ${ }^{3}$ Department of Pathology, University of Florida, Gainesville, FL, USA; ${ }^{4}$ Department of Radiology, Johns Hopkins University School of Medicine, Baltimore, MD, USA and ${ }^{5}$ Department of Medical Oncology, Johns Hopkins University School of Medicine, Baltimore, MD, USA

\begin{abstract}
Hepatocellular carcinomas exhibit heterogeneous morphologies by routine light microscopy. Although some morphologies represent insignificant variations in growth patterns, others may represent unrecognized subtypes of hepatocellular carcinoma. Identification of these subtypes could lead to separation of hepatocellular carcinomas into discrete groups with unique underlying genetic changes, prognosis, or therapeutic responses. In order to identify potential subtypes, two pathologists independently screened a cohort of 219 unselected hepatocellular carcinoma resection specimens and divided cases into potential subtypes. One of these promising candidate subtypes was further evaluated using histological and molecular techniques. This subtype was characterized by a unique and consistent set of histological features: smooth chromophobic cytoplasm, abrupt focal nuclear anaplasia (small clusters of tumor cells with marked nuclear anaplasia in a background of tumor cells with bland nuclear cytology), and scattered microscopic pseudocysts-we designate this variant as 'chromophobe hepatocellular carcinoma with abrupt anaplasia'. Thirteen cases were identified (6\% of all hepatocellular carcinomas), including 6 men and 7 women with an average age of 61 years. Six cases occurred in cirrhotic livers. Serum AFP was elevated in 6 out of 10 cases. There were a variety of underlying liver diseases, but cases were enrichment for chronic hepatitis $B, P=0.006$. Interestingly, at the molecular level, this variant was strongly associated with the alternative lengthening of telomere (ALT) phenotype by telomere FISH. ALT is a telomerase-independent mechanism of telomere maintenance and is found in approximately $8 \%$ of unselected hepatocellular carcinomas. In contrast, $11 / 12$ (92\%) of the cases of chromophobe hepatocellular carcinoma with abrupt anaplasia were ALT-positive. In summary, we propose that chromophobe hepatocellular carcinoma with abrupt anaplasia represents a new subtype of hepatocellular carcinoma with unique morphological and molecular features.

Modern Pathology (2013) 26, 1586-1593; doi:10.1038/modpathol.2013.68; published online 3 May 2013
\end{abstract}

Keywords: ALT; hepatocellular carcinoma; telomere

Hepatocellular carcinoma is the most common primary liver cancer and is a significant cause of morbidity and mortality. Hepatocellular carcinoma is the sixth most common cancer diagnosis and the

Correspondence: Dr MS Torbenson, MD, Department of Pathology, Johns Hopkins University School of Medicine, 1503 E Jefferson, Room B314, Baltimore, MD 21231, USA.

E-mail: mtorben@jhmi.edu

Received 30 July 2012; revised 9 January 2013; accepted 9 January 2013; published online 3 May 2013 third leading cause of cancer death worldwide, with approximately 600000 deaths per year. ${ }^{1,2}$ The overall prognosis for individuals with hepatocellular carcinoma is poor.

The histological findings in hepatocellular carcinoma have been extensively described in the pathology literature. In this context, a wide variety of histological patterns are recognized as consistent with a diagnosis of hepatocellular carcinoma. Some patterns are further recognized as unique types of 
primary liver carcinoma, such as the histological findings in fibrolamellar carcinoma. ${ }^{3}$ Yet, even within cases diagnosed as typical hepatocellular carcinoma, there are a wide range of morphological findings and clinical outcomes. This led us to consider the possibility that other morphological variants remain to be described. Although some morphologies likely represent variations in growth patterns with little clinical or biological significance, other morphologies may represent unrecognized variants of hepatocellular carcinoma with distinct clinical and/or molecular features. Identification of these variants could lead to separation of hepatocellular carcinoma into discrete groups with unique underlying genetic changes, epigenetic changes, prognosis, or therapeutic response. This would likely deepen our understanding of the biology of hepatocellular carcinoma and possibly alter clinical management.

\section{Materials and methods}

\section{Identification of Variants}

A cohort was collected of 219 unselected hepatocellular carcinomas from Johns Hopkins University that had been resected during the time period of 1985 to 2011. All cases were full resections for curative intent or were liver transplants. These 219 cases represent $61 \%$ of all hepatocellular carcinoma resections from this time period. This set of cases was first evaluated for possible subtypes by a GI/ liver pathologist (MST), and a case list of possible subtypes was generated. Next, a second GI/liver pathologist (MRA) independently evaluated the same cases and generated an independent list of candidate morphological subtypes. The two lists were then compared and discussed. One of the promising candidate morphologies was then selected for further studies. Electronic medical records were reviewed by an oncologist specializing in hepatocellular carcinoma (DC). In addition, the available radiology studies were reviewed by a radiologist specializing in imaging of the liver (IRK).

\section{Analysis of Alternative Lengthening of Telomere}

After identification of a potential subtype of hepatocellular carcinoma (provisionally called chromophobe hepatocellular carcinoma with abrupt anaplasia), we retrospectively looked back at data sets from our laboratories to see if this variant of hepatocellular carcinoma had been included in previous studies, before they were recognized as a unique subtype. This retrospective analysis showed that six cases of chromophobe hepatocellular carcinoma with abrupt anaplasia had been included in a previous study that examined the frequency of the alternative lengthening of telomere (ALT) phenotype in a broad survey of carcinomas from major organ systems. ${ }^{4}$ ALT is a telomerase-independent mechanism of maintaining telomeres ${ }^{5}$ that can be seen in a broad range of human malignances. ${ }^{4,6}$ In this prior study, $8 \%$ of unselected hepatocellular carcinomas, analyzed in tissue microarray format, demonstrate ALT by telomere-specific FISH. ${ }^{4}$ However, surprisingly, in retrospect, five of the six cases of chromophobe hepatocellular carcinoma with abrupt anaplasia included on the tissue microarrays were ALT-positive. This finding suggested that chromophobe hepatocellular carcinomas with abrupt anaplasia were strongly enriched for the ALT phenotype. To investigate this further, telomerespecific FISH was performed using previously described methods on all cases of chromophobe hepatocellular carcinoma with abrupt anaplasia. Briefly, blocks from the surgical resections were cut at $5 \mu \mathrm{m}$, deparaffinized, underwent antigen retrieval by steaming for $20 \mathrm{~min}$ in citrate buffer (Vector Laboratories), and were then hybridized with a Cy3-labeled peptide nucleic acid (PNA) probe complementary to the mammalian telomere repeat sequence ( $\left(\mathrm{N}\right.$ terminus to $\mathrm{C}$ terminus) $5^{\prime}$ CCCTAACCCTAACCCTAA-3'). Human centromeric DNA was also labeled as a positive control for hybridization efficiency using a FITC-labeled PNA probe (5'-ATTCGTTGGAAACGGGA-3'; CENP-Bbinding sequence). Nuclei were counterstained with DAPI, viewed by fluorescence microscopy, and assessed for the ALT phenotype. ALT is readily identified as large, ultrabright aggregates of telomeric DNA that are unique to ALT-positive tumor cell populations. Tumors were classified as ALT-positive when $\geqslant 1 \%$ of the neoplastic cells displayed the abnormal ALT-associated telomeric DNA foci, as per following previously published criteria. $^{4,7}$ Necrotic areas were excluded from analysis.

\section{Sequencing of ATRX, DAXX, and H3F3A}

Two cases of chromophobe hepatocellular carcinoma with abrupt anaplasia had frozen tissues available. Mutations in ATRX, DAXX, and H3F3A have all been associated with the ALT phenotype. ${ }^{7,8}$ Genomic DNA was isolated from fresh-frozen tumor and paired non-neoplastic liver tissues, and all exons of ATRX, DAXX, and $H 3 F 3 A$ were amplified and directly Sanger sequenced.

\section{Immunohistochemistry}

Mutations in TP53 have also been linked to the ALT phenotype. ${ }^{9}$ To investigate if the cases of chromophobe hepatocellular carcinoma with abrupt anaplasia had abnormal nuclear accumulation of p53 protein, tumor samples were analyzed by immunohistochemistry. Routine immunostaining for P53 was performed after antigen retrieval on $5 \mu \mathrm{m}$ sections (Ventana; clone BP53-11). 


\section{Results}

Independent evaluation of a cohort of 219 unselected hepatocellular carcinomas by two pathologists revealed a subset of 13 cases with morphological findings, suggesting that they represent a new subtype of hepatocellular carcinoma. This subtype was characterized by a unique and consistent set of shared histological features: smooth chromophobic cytoplasm, abrupt focal nuclear anaplasia (small clusters of tumor cells with marked nuclear atypia in a background of tumor cells with bland, round nuclei, and inconspicuous nucleoli), and scattered microscopic pseudocysts (Figure 1).

In three cases of chromophobe hepatocellular carcinoma with abrupt anaplasia, the cytoplasm was more eosinophilic in areas of the tumor, but there were other areas of more typical chromophobe appearance, and the nuclear features and pseudocysts were helpful in identifying the tumors as chromophobe hepatocellular carcinomas with abrupt anaplasia. Of the tumors with more eosinophilic cytoplasm, two of the cases had prior embolic therapy and one was cholestatic, and it is possible that these events may have affected the cytoplasmic appearances. Of the 13 cases of chromophobe hepatocellular carcinoma with abrupt anaplasia, 3 had minimal macrovesicular steatosis and 2 had mild macrovesicular steatosis.

The chromophobe hepatocellular carcinomas with abrupt anaplasia averaged $5.4 \pm 3.8 \mathrm{~cm}$ in greatest dimension (Table 1). Angiolymphatic invasion was seen in three cases and in each was extensive. In 12 cases, the tumor was composed entirely of the morphology typical of chromophobe hepatocellular carcinomas with abrupt anaplasia. In one case, the chromophobe hepatocellular carcinoma with abrupt anaplasia was a distinct nodule, whereas other separate and distant nodules of hepatocellular carcinoma in the same liver specimen lacked the morphological features and instead showed typical hepatocellular carcinomas with trabecular growth patterns. Pseudocapsules of compressed fibrous tissue were present in 11/13 cases and were often thick and irregular. The tumors often had bands of intra-tumoral fibrosis that divided the tumor into smaller sections (9/13 cases). Eight cases had areas of geographic tumor necrosis, four of which also had histories of preoperative embolic therapy.

The background liver showed cirrhosis in six cases, bridging fibrosis in one case, portal fibrosis in one case, and no fibrosis in five cases (Table 1). Chromophobe hepatocellular carcinomas with abrupt anaplasia had a higher frequency of HBV as the underlying cause of liver disease when compared with typical hepatocellular carcinomas (Pearson's $\chi^{2}$-test, $P=0.006$; Fisher's exact test, $P=0.013$ ). Overall, seven cases occurred in the setting of chronic viral hepatitis (six with hepatitis B and one with hepatitis $\mathrm{C}$ ), one in the setting of alcoholic cirrhosis, one with cryptogenic cirrhosis, and one arose in a hepatic adenoma with marked fatty change. One additional case arose in the setting of no known underlying liver disease. Insufficient clinical information was available on the remaining two cases to determine a cause of liver disease. Preoperative alpha-feto protein levels were markedly elevated (>400 ng/ $\mathrm{ml}$, normal $<10 \mathrm{ng} / \mathrm{ml}$ ) in 6 out of 10 patients with available alpha-feto protein levels. The usual male enrichment for HCC was not evident in chromophobe hepatocellular carcinomas with abrupt anaplasia, though the findings were not statistically significant (Table 1).

\section{Mimics of Chromophobe Hepatocellular Carcinomas with Abrupt Anaplasia}

When examining H\&E sections, one potential mimic of chromophobe hepatocellular carcinomas with abrupt anaplasia are cases with prominent pseudocysts (Figure 2). However, most cases with pseudocysts are not chromophobe hepatocellular carcinomas with abrupt anaplasia, as they lack the remaining features of chromophobe cytoplasm and overall bland nuclei with patchy areas of striking nuclear anaplasia.

A more challenging mimic of chromophobe hepatocellular carcinomas with abrupt anaplasia are some cases of clear cell hepatocellular carcinoma, in particular those that have patchy areas of anaplasia (Figure 2). However, clear cell carcinomas with anaplasia tend to have distinctly different nuclei outside the areas of anaplasia. In contrast to the cytologically bland nuclei with inconspicuous nucleoli of chromophobe hepatocellular carcinomas with abrupt anaplasia, clear cell hepatocellular carcinomas tend to have more vacuolated nuclei, often with prominent nucleoli. In addition, the cytoplasmic tinctorial qualities are different and can be helpful in many cases.

\section{Immunohistochemistry}

Immunostains performed at the time of initial case sign out showed negative staining for EMA $(2 / 2$ cases), chromogranin (2/2), cytokeratin AE1/3 (3/3), and monoclonal CEA (3/3). Polyclonal CEA showed positive cytoplasmic and canalicular staining in $3 / 3$ cases. Ki-67 in two cases showed $30 \%$ and $40 \%$ tumor nuclear labeling, respectively (estimated to the nearest $10 \%$ ).

Because of the possible links between the ALT phenotype and p53 abnormalities, we also performed p53 immunostaining on 12 cases. Of these 12 cases, 4 showed nuclear labeling for p53: 3 cases showed moderate-to-strong staining in at least $50 \%$ of tumor cells, whereas 1 case showed weak focal staining in $<10 \%$ of tumor cells.

\section{Imaging Findings}

All available preoperative cross-sectional imaging studies were reviewed. Six of the 13 reported cases 

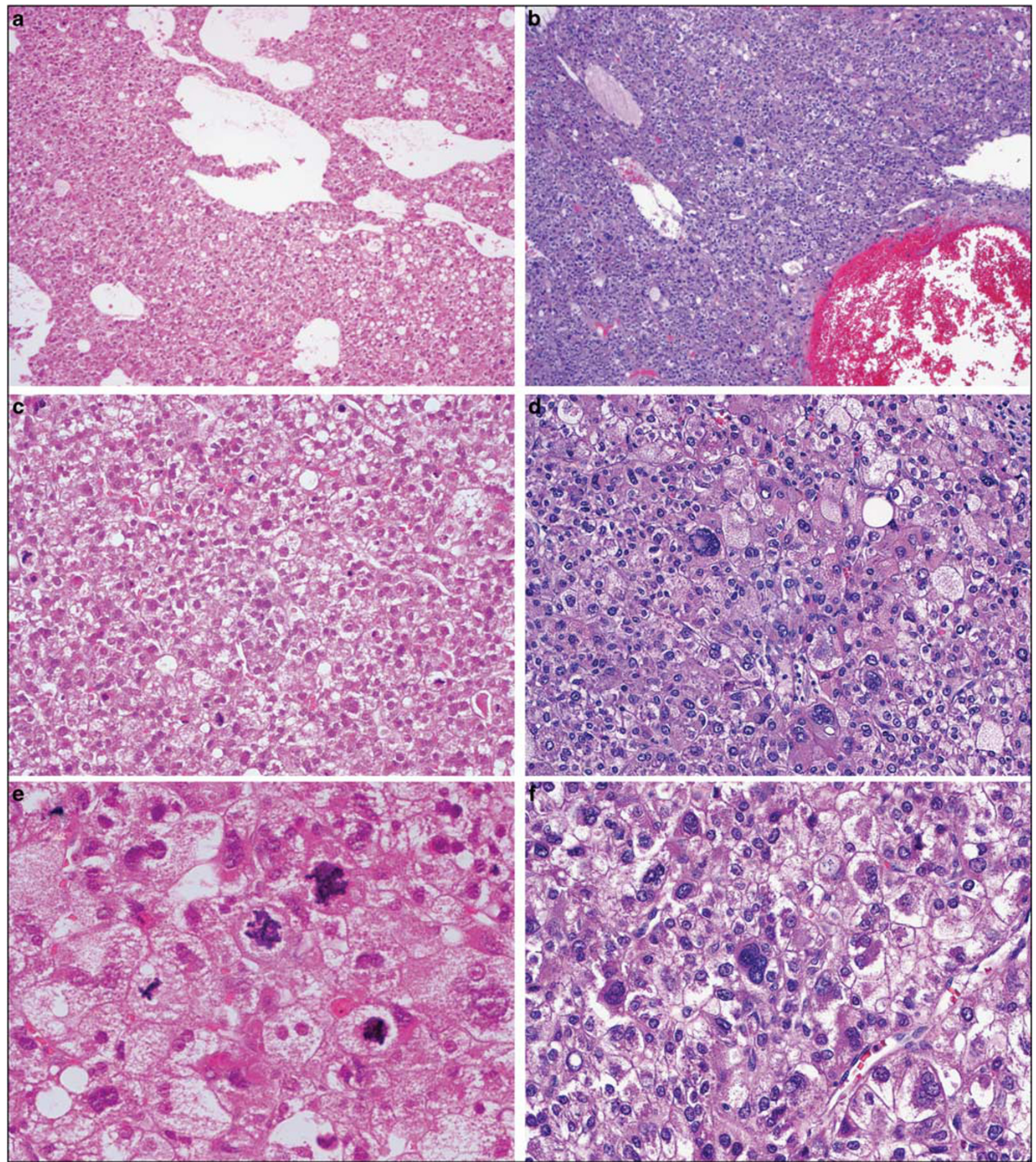

Figure 1 Chromophobe hepatocellular carcinomas with abrupt anaplasia. Two separate cases are illustrated, with the first case in the left column of panels and the second in the right column of panels. Pseudocysts are evident in both cases (a, b; H\&E, original magnification $\times 40$ ). The cytoplasm of the tumor cells have a 'chromophobe appearance' where the staining qualities of the tumor cytoplasm are not as deep as seen in typical hepatocellular carcinomas (c, $\mathbf{d}$; H\&E, original magnification $\times 100$ ). The cytoplasm can be either slightly eosinophilic (c) or basophilic (d). The nuclei of most tumor cells are small and round with small inconspicuous nucleoli. However, cells in small irregularly distributed clusters show marked nuclear anaplasia (e, f; H\&E, original magnification $\times 160$ ).

had MRI (5) or CT (1) studies. In five of the six patients, the mass was hypervascular in the hepatic arterial phase and demonstrated washout in the portal venous phase, features that are typical for hepatocellular carcinoma. In the remaining patient, the mass was hypervascular in the arterial phase and demonstrated progressive enhancement in the portal venous phase, with large areas of central 
necrosis. In the five patients who had preoperative MRI, the mass was slightly hypointense on T1weighted images and slightly hyperintense of T2weighted images. None of these five patients demonstrated intra or extra cellular fat on MRI. Five of six tumors had a thick pseudocapsule. None of the lesions demonstrated central calcifications, and adenopathy was absent in all cases.

\section{ALT Phenotype}

Previous analyses of a small number of cases of chromophobe hepatocellular carcinomas with abrupt anaplasia (before they were recognized as such) found that they were positive for the ALT phenotype. ${ }^{4}$ To confirm this association, we

Table 1 Demographic and clinical findings

\begin{tabular}{|c|c|c|c|}
\hline Observation & $\begin{array}{l}\text { Chromophobe } \\
\text { hepatocellular } \\
\text { carcinoma } \\
\text { with abrupt } \\
\text { anaplasia }\end{array}$ & $\begin{array}{c}\text { Hepatocellular } \\
\text { carcinoma } \\
\text { with typical } \\
\text { morphology }\end{array}$ & $\begin{array}{c}\text { Pearson's } \\
\chi^{2} \\
\text { P-value }\end{array}$ \\
\hline Total cases & 13 & 206 & \\
\hline Age, mean \pm s.d. & $60 \pm 8$ & $56 \pm 16$ & 0.34 \\
\hline $\begin{array}{l}\text { Gender } \\
\text { Male } \\
\text { Female }\end{array}$ & $\begin{array}{l}46 \% \\
54 \%\end{array}$ & $\begin{array}{l}66 \% \\
34 \%\end{array}$ & 0.061 \\
\hline $\begin{array}{l}\text { Cirrhosis (\%) } \\
\text { Elevated serum } \\
\text { AFP (\%) }\end{array}$ & $\begin{array}{l}46 \%^{\mathrm{a}} \\
50 \%{ }^{\mathrm{c}}\end{array}$ & $\begin{array}{l}48 \% \mathrm{~b} \\
35 \%^{\mathrm{d}}\end{array}$ & $\begin{array}{l}0.67 \\
0.327\end{array}$ \\
\hline $\begin{array}{l}\text { Tumor size (cm) } \\
\text { Chronic HCV (\%) } \\
\text { Chronic HBV (\%) }\end{array}$ & $\begin{array}{c}5.7 \pm 4.4 \\
8 \% \mathrm{e}^{\mathrm{e}} \\
46 \%\end{array}$ & $\begin{array}{l}5.4 \pm 3.8 \\
30 \% \mathrm{f} \\
16 \%\end{array}$ & $\begin{array}{l}0.99 \\
0.073 \\
0.006\end{array}$ \\
\hline
\end{tabular}

${ }^{\mathrm{a}}$ Information was available on 11 cases.

bInformation was available on 194 cases.

${ }^{\mathrm{C}}$ Information was available on 12 cases.

${ }^{\mathrm{d}}$ Information was available on 150 cases.

e Information was available on 11 cases.

f Information was available on 179 cases.

Figure 2 Mimics of chromophobe hepatocellular carcinomas with abrupt anaplasia. Pseudocysts are a regular feature of chromophobe hepatocellular carcinomas with abrupt anaplasia, but most cases of hepatocellular carcinoma with pseudocysts will not be chromophobe hepatocellular carcinomas with abrupt anaplasia. (a) A hepatocellular carcinoma with pseudocysts that is not a chromophobe hepatocellular carcinoma with abrupt anaplasia (H\&E, original magnification $\times 64)$. (b) A hepatocellular carcinoma with cytoplasmic findings that somewhat mimic the chromophobe cytoplasm of chromophobe hepatocellular carcinomas with abrupt anaplasia, but this tumor lacks pseudocysts, sudden anaplasia, and has prominent nucleoli, all features inconsistent with chromophobe hepatocellular carcinoma with abrupt anaplasia $(\mathrm{H} \& \mathrm{E}$, original magnification $\times 160)$. (c) A clear cell hepatocellular carcinoma with areas of marked anaplasia. This morphological pattern can be a strong mimic of chromophobe hepatocellular carcinomas with abrupt anaplasia, but the nuclear changes in most clear cell hepatocellular carcinoma (irregular vesiculated nuclei with prominent nucleoli) are sufficient to distinguish this mimic from chromophobe hepatocellular carcinomas with abrupt anaplasia (H\&E, original magnification $\times 160$ ). assessed the ALT phenotype in all cases of chromophobe hepatocellular carcinomas with abrupt anaplasia. ALT status was determined using telomere-specific FISH, with ALT-positive tumors showing ultrabright intranuclear foci of telomere FISH signals in $\geq 1 \%$ of tumor cells. The ALT phenotype was present in 11 of 13 chromophobe hepatocellular carcinomas with abrupt anaplasia (Figure 3). The ultrabright intranuclear foci of





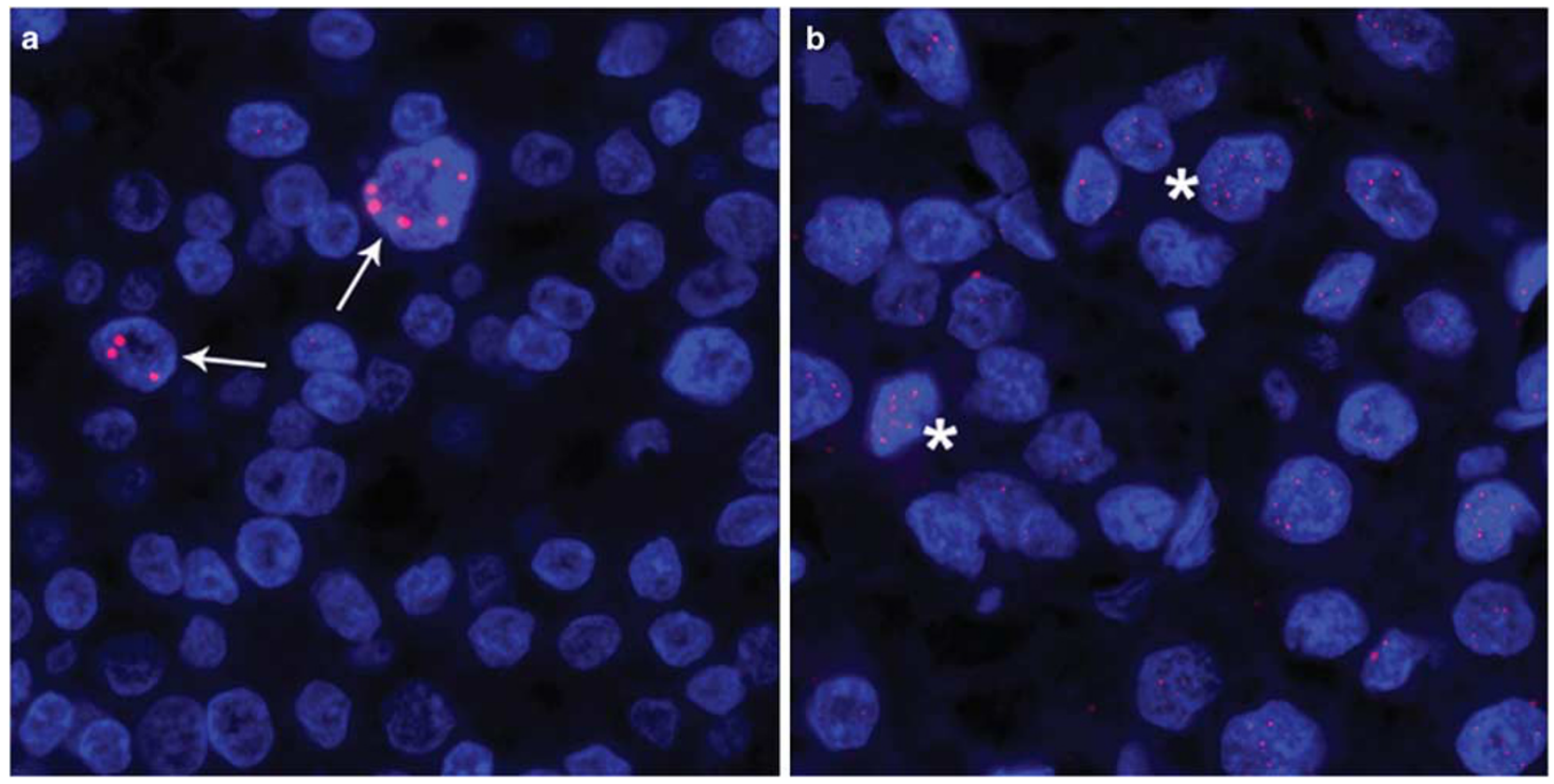

Figure 3 Telomere FISH. Telomere-specific FISH demonstrating ALT-positive and ALT-negative tumors. (a) Representative example of a chromophobe hepatocellular carcinoma with abrupt anaplasia demonstrating the ALT phenotype. The arrows indicate the distinctive large, ultrabright, intranuclear foci of telomere FISH signals that mark the ALT-associated telomeric foci. (b) Representative example of an ALT-negative hepatocellular carcinoma with typical morphology. This tumor lacks the morphology of chromophobe hepatocellular carcinoma with abrupt anaplasia. The asterisks indicate robust telomere signals present in the tumor cells. Original magnification $\times 400$. Because the telomere signals in the ALT-positive case quickly become saturated, the cy3 exposure time in a was $200 \mathrm{~ms}$, whereas in b, it was $600 \mathrm{~ms}$.

telomeric DNA did not correlate with the areas of marked anaplasia. In one additional case, the tumor showed a few ultrabright telomere FISH signals seen with the ALT phenotype, but the number of foci did not reach the threshold to be classified as ALTpositive. The one negative case had relatively little viable tumor on the stained section.

Of note, not all hepatocellular carcinomas with ALT positivity will be chromophobe hepatocellular carcinomas with abrupt anaplasia. Retrospective histological review of previously published ALTpositive hepatocellular carcinomas from this instution $^{4}$ indicated that $2.4 \%(2 / 85)$ of hepatocellular carcinomas were ALT-positive after excluding those cases that had the morphological features of chromophobe hepatocellular carcinomas with abrupt anaplasia, at least when evaluated on tissue microarrays.

\section{Sequencing of ATRX, DAXX, and H3F3A}

Previous studies have identified somatic mutations in the ATRX, DAXX, and H3F3A genes in neoplasms with the ALT phenotype, including pancreatic neuroendocrine tumors and primary central nervous system neoplasms., ${ }^{7,8}$ Thus, we assessed the sequences of these genes in two cases for which fresh-frozen tumor and paired normal tissue were available. Direct sequencing of all coding exons of $A T R X, D A X X$, and H3F3A revealed no somatic mutations.

\section{Discussion}

In this study, we describe chromophobe hepatocellular carcinomas with abrupt anaplasia and propose that they represent a distinct subtype of hepatocellular carcinoma. Chromophobe hepatocellular carcinomas with abrupt anaplasia have consistent and unique histological findings and are strongly enriched for the ALT telomere-maintenance phenotype. They arise in cirrhotic and non-cirrhotic livers and are associated with a variety of underlying liver disease settings. In this single center study, they make up $6 \%$ of hepatocellular carcinomas.

Chromophobe hepatocellular carcinomas with abrupt anaplasia was originally identified through recognition of its unique morphological features by two independent pathologists-these features include chromophobic cytoplasm, abrupt anaplasia, and pseudocysts. In this study, we show that this variant has both a unique morphology and a distinct molecular mechanism with respect to telomere maintenance. Sustained telomere maintenance is a feature of many human cancers. Although most cancers $(\sim 90 \%)$, including hepatocellular carcinomas, maintain their telomeres through telomerase activity, a subset of cancers utilize a telomeraseindependent mechanism termed ALT, which is dependent on homologous recombination. ${ }^{5}$ This study represents the first report of a high prevalence of the ALT phenotype in a morphologically distinct carcinoma subtype. The dependence 
on homologous recombination in these tumors may allow development of targeted therapeutics and its use as a predictive biomarker. In this cohort, the wide range in therapies and the lack of sufficiently detailed follow-up information did not permit us to examine the prognostic significance of chromophobe hepatocellular carcinomas with abrupt anaplasia, and further studies with extended clinical follow-up will be necessary to determine the overall prognosis.

The ALT phenotype has been linked to somatic mutations in the ATRX and DAXX genes. This association has been found in multiple tumor types, including pancreatic neuroendocrine tumors and primary central nervous system neoplasms. In addition, somatic mutations in the H3F3A gene have been reported in pediatric gliomas with the ALT phenotype. ${ }^{8}$ The proteins encoded by the ATRX and $D A X X$ genes form a heterodimer and have multiple cellular roles, including chromatin remodeling. ${ }^{10}$ The histone protein encoded by H3F3A is incorporated into heterochromatin by the ATRX-DAXX protein complex. ${ }^{11}$ Therefore, mutations in these genes are thought to play a role in the development of the ALT phenotype in certain tumor types. ${ }^{7}$ The lack of mutations in $A T R X$, $D A X X$, and $H 3 F 3 A$ in chromophobe hepatocellular carcinomas with abrupt anaplasia suggests the possibility of a different genetic alteration underlying the ALT phenotype in these tumors. As one possible example, Hepatitis B can interrupt the telomerase reverse transcriptase gene TERT, ${ }^{12}$ which could disrupt normal telomere-maintenance networks and potentially select for the ALT phenotype.

All of the chromophobe hepatocellular carcinomas with abrupt anaplasia were readily identified as hepatocellular carcinomas at the time of original surgical pathology review, and their proposed identification as a unique subtype is not because they pose a diagnostic challenge. Instead, their recognition is of value because (1) this subtype represents one of the first known morphological subtypes of carcinoma that are highly enriched for the ALT phenotype, (2) chromophobe hepatocellular carcinomas with abrupt anaplasia are predicted to have different gene-expression patterns than typical hepatocellular carcinomas, an important consideration when searching for hepatocellular carcinoma biomarkers and novel therapeutic targets, and (3) finally, chromophobe hepatocellular carcinomas with abrupt anaplasia may respond differently than typical hepatocellular carcinomas to standard therapy. In regards to the latter point, the recognition of distinct subtypes of hepatocellular carcinoma, some of which may respond differently to therapy, is likely to be increasingly important as new drugs are developed to treat hepatocellular carcinomas. ${ }^{13}$ Just as combining fibrolamellar carcinomas with typical hepatocellular carcinomas in a clinical or molecular biology study would be highly likely to obscure important molecular, clinical, and treatment outcome findings, we propose that chromophobe hepatocellular carcinomas with abrupt anaplasia should undergo subset analysis in studies that focus on the molecular biology, biomarker development, or treatment response of hepatocellular carcinomas.

In the remainder of this discussion, we address the rationale and approach to subtyping hepatocellular carcinomas. Subdividing hepatocellular carcinoma makes sense when a subtype can be identified by routine surgical pathology and when its recognition as a subtype conveys clinically meaningful information, with the realization that the clinically meaningful information may take many forms, including prognostic information, etiological considerations, and response to therapy. It seems likely that the expansion of therapeutic options for the treatment of hepatocellular carcinoma may enhance the importance of recognizing subtypes of hepatocellular carcinoma. Indeed, the majority of recent clinical trials of targeted chemotherapeutic agents in unselected hepatocellular carcinoma have proven disappointing, ${ }^{14}$ highlighting the need to enrich treatment populations with patients more likely to benefit from a particular agent or combination of agents, as has been shown in other tumor types, such as melanoma and colorectal cancer.

In general, it appears that an ideal subtype of hepatocellular carcinoma should possess four distinct elements: (1) a unique H\&E morphology that can be recognized by routine surgical pathology; (2) sensitive and specific immunostains to confirm the H\&E impression; (3) unique molecular features; and (4) clinical correlates. It is not the case that all subtypes of hepatocellular carcinoma will have detailed information in all of these four categories from the outset. As a practical point, we propose that at least two of these categories should be sufficiently well defined as a minimum for recognizing a unique type of hepatocellular carcinoma. In this regard, there are many histological findings in hepatocellular carcinomas that by themselves may be striking, but are not sufficient to be termed a unique subtype of hepatocellular carcinoma, as they do not fulfill at least two of the criteria for a subtype of hepatocellular carcinoma.

To expand on the four elements that define a subtype of hepatocellular carcinoma, the first element, a unique $\mathrm{H} \& \mathrm{E}$ morphology is important because routine surgical pathology is currently the first line of tumor evaluation in many cases. Thus, potential hepatocellular carcinoma variants can be recognized in real time at the point of medical intervention. Ideally, a hepatocellular carcinoma that is recognized as a potential subtype would then be confirmed by immunostains. Confirmation of potential tumor subtypes with immunostains is important because H\&E findings are not always sufficient. As an illustration of this point, fibrolamellar carcinomas have distinctive $\mathrm{H} \& \mathrm{E}$ findings 
that have been recognized by pathologists for decades, but it is not uncommon for fibrolamellar carcinomas to be misdiagnosed. However, immunostain markers for fibrolamellar carcinomas (CD68 and CK7) have now been reported that allow confirmation of the $\mathrm{H} \& \mathrm{E}$ findings, providing greater confidence in the histological diagnosis of fibrolamellar carcinoma. ${ }^{15}$ Thus, when identifying a subtype of hepatocellular carcinoma, ideally the $\mathrm{H} \& \mathrm{E}$ findings would strongly suggest a particular subtype of hepatocellular carcinoma, and immunostains would be used on this subset to confirm the diagnosis. At this point, chromophobe hepatocellular carcinomas with abrupt anaplasia do not have an immunostain for confirmation, but telomere-specific FISH for the ALT phenotype could partly serve this role.

The third element of the definition of a subtype of hepatocellular carcinoma, unique molecular features, will not always be known, even when a subtype of hepatocellular carcinoma is widely accepted. Fibrolamellar carcinomas again serve as useful illustration, as unique molecular biology findings remain largely unknown. ${ }^{3}$ In contrast, chromophobe hepatocellular carcinomas with abrupt anaplasia have a strong association with the ALT phenotype. The final element in the definition of a subtype of hepatocellular carcinoma, that of clinical correlates, will likely take different forms depending on the subtype of hepatocellular carcinoma. Continuing with the example of fibrolamellar carcinomas, key clinical findings include a young age at presentation, equal gender distribution, no known underlying liver disease, tumor calcifications on imaging studies, and increased frequency of lymph node metastases. ${ }^{3}$ Chromophobe hepatocellular carcinomas with abrupt anaplasia appear to have a more even gender distribution and to be enriched for cases with $\mathrm{HBV}$ as the underlying cause of liver disease, but these findings await confirmation from further studies.

One of the limits of this study is that we have not shown that chromophobe hepatocellular carcinomas with abrupt anaplasia can be identified in liver biopsy specimens. This clearly is an important goal and one that will need to be addressed by future studies specifically focused on that question. It seems likely to us that identification of a robust immunostain that can be used to confirm the H\&E impression of a chromophobe hepatocellular carcinoma with abrupt anaplasia will have a critical role in facilitating biopsy diagnosis.

In summary, these studies describe a novel variant of hepatocellular carcinoma-chromophobe hepatocellular carcinomas with abrupt anaplasia, which possess unique and consistent morphological findings (chromophobic cytoplasm, abrupt anaplasia, and pseudocysts), as well as a distinct molecular abnormality (ALT phenotype). Chromophobe hepatocellular carcinomas with abrupt anaplasia appear to have a more equal gender distribution and are enriched for chronic hepatitis B as the underlying cause of liver disease. The recognition of chromophobe hepatocellular carcinomas with abrupt anaplasia represents an important step in the separation of hepatocellular carcinoma into biologically and clinically meaningful variants.

\section{Disclosure/conflict of interest}

The authors declare no conflict of interest.

\section{References}

1 Forner A, Llovet JM, Bruix J. Hepatocellular carcinoma. Lancet 2012;379:1245-1255.

2 Venook AP, Papandreou C, Furuse J, et al. The incidence and epidemiology of hepatocellular carcinoma: a global and regional perspective. Oncologist 2010;15(Suppl 4):5-13.

3 Torbenson M. Review of the clinicopathologic features of fibrolamellar carcinoma. Adv Anat Pathol 2007;14: 217-223.

4 Heaphy CM, Subhawong AP, Hong SM, et al. Prevalence of the alternative lengthening of telomeres telomere maintenance mechanism in human cancer subtypes. Am J Pathol 2011;179:1608-1615.

5 Cesare AJ, Reddel RR. Alternative lengthening of telomeres: models, mechanisms and implications. Nat Rev Genet 2010;11:319-330.

6 Henson JD, Reddel RR. Assaying and investigating alternative lengthening of telomeres activity in human cells and cancers. FEBS Lett 2010;584:3800-3811.

7 Heaphy CM, de Wilde RF, Jiao Y, et al. Altered telomeres in tumors with ATRX and DAXX mutations. Science 2011;333:425.

8 Schwartzentruber J, Korshunov A, Liu XY, et al. Driver mutations in histone H3.3 and chromatin remodelling genes in paediatric glioblastoma. Nature 2012;482: 226-231.

9 Chen YJ, Hakin-Smith V, Teo M, et al. Association of mutant TP53 with alternative lengthening of telomeres and favorable prognosis in glioma. Cancer Res 2006;66:6473-6476.

10 Xue Y, Gibbons R, Yan Z, et al. The ATRX syndrome protein forms a chromatin-remodeling complex with Daxx and localizes in promyelocytic leukemia nuclear bodies. Proc Natl Acad Sci USA 2003;100:10635-10640.

11 Lewis PW, Elsaesser SJ, Noh KM, et al. Daxx is an H3.3-specific histone chaperone and cooperates with ATRX in replication-independent chromatin assembly at telomeres. Proc Natl Acad Sci USA 2010;107: 14075-14080.

12 Sung WK, Zheng H, Li S, et al. Genome-wide survey of recurrent HBV integration in hepatocellular carcinoma. Nat Genet 2012;44:765-769.

13 Schirmacher P, Bedossa P, Roskams T, et al. Fighting the bushfire in HCC trials. J Hepatol 2011;55:276-277.

14 Kudo M. Current status of molecularly targeted therapy for hepatocellular carcinoma: clinical practice. Int J Clin Oncol 2010;15:242-255.

15 Ross HM, Daniel HD, Vivekanandan P, et al. Fibrolamellar carcinomas are positive for CD68. Mod Pathol 2011;24:390-395. 